\title{
Recommended adequate exercise for diabetic patients in response to new lifestyle manner with corona era for Global health
}

\begin{abstract}
Crucial problems of Coronavirus disease 2019 (COVID-19) are found worldwide. Elderly people and diabetic patients tend to show less activity or exercise. "Exercise is Medicine" has been known by American College of Sports Medicine (ACSM). From 24 articles with 10948 COVID-19 cases, the prevalence (odds ratio, OR) showed diabetes in 10.0\% (2.61), Cardiovascular disease (CVD) in $8.0 \%$ (4.81), hypertension in $20.0 \%$ (2.84), chronic pulmonary disease in $3.0 \%$ (3.83). Out of 7337 cases of COVID-19, there were 952 Type 2 Diabetes Mellitus (T2DM) patients. The mortality of diabetes vs non-diabetes was $7.8 \%$ vs $2.7 \%$, respectively (HR 1.49). Regarding these diabetics, cases with max glucose below $180 \mathrm{mg} / \mathrm{dL}$ showed $1.1 \%$ of mortality, whereas case with above $180 \mathrm{mg} / \mathrm{dL}$ showed $11.0 \%$. People's lifestyles are changing due to corona infections, and this article is expected to become useful reference for adequate exercise leading to global health.
\end{abstract}

Volume 9 Issue 4 - 2020

\author{
Hiroshi Bando 1,2 \\ 'Medical Research, Tokushima University, Japan \\ ${ }^{2}$ Chairman, Shikoku Island Division, Integrative Medicine Japan, \\ Japan
}

Correspondence: Hiroshi Bando, MD, PhD, FACP, Medical Research, Tokushima University, Nakashowa I-6I,Tokushima 770-0943, Japan, Tel +81-90-3187-2485,

Email pianomed@bronze.ocn.ne.jp

Received: July 30, 2020 | Published: August II, 2020

Keywords: exercise is medicine, frequency, intensity, time, type, volume

Abbreviations: COVID-19, coronavirus disease 2019; ACSM, American College of Sports Medicine; LTPA, leisure-time physical activity; PAGA, Physical Activity Guidelines for Americans

\section{Opinion}

There has been recently medical and social problem of Coronavirus disease 2019 (COVID-19) worldwide. ${ }^{1}$ Some topics on exercise habit for the diabetics would be described in this article. There is a famous phrase "Exercise is Medicine". ${ }^{2}$ In fact, exercise can prevent or treat multiple diseases and conditions. American College of Sports Medicine (ACSM) emphasizes the importance of exercise prescribing just like oral medicine, and recommends that physical activity would be assessed for all occasions and for all. ${ }^{3}$ The purpose is to progress the health, and to improve physical fitness such as body endurance, muscular strength/endurance, and flexibility. The specific prescription content is to make the definition of six items (FITT-VP). ${ }^{4}$ They include frequency, intensity, time, type, volume and progression. ${ }^{3}$

From psychological point of view, elderly people feel anxious for the corona infection. Then, they tend to keep still at home. Continuing this lifestyle, they will have some risks for muscle atrophy, gait weakness leading to frail/sarcopenia, and for elevated blood glucose, triglyceride and blood pressure leading to atherosclerotic cardiovascular disease (ASCVD). There was the health ABC study cohort for 70-79 years old. Mid-thigh muscle cross-sectional area (CSA) was declined two times faster in diabetic than non-diabetic counterparts. ${ }^{5}$ When these situations persist, there will be elevated risk for frail, sarcopenia and dementia.

COVID-19 has been involved in mortality and morbidity across the world. The efficacy of chronic comorbid diseases on COVID-19 was studied in databases. ${ }^{6}$ Totally 24 articles with 10948 cases were analyzed. The results of prevalence (plus odds ratio, OR) showed diabetes in $10.0 \%$ (OR 2.61), Cardiovascular disease (CVD) in $8.0 \%$ (OR 4.81), hypertension in $20.0 \%$ (OR 2.84), chronic pulmonary disease in $3.0 \%$ (OR 3.83)
Out of 7337 cases of COVID-19, there were 952 T2DM patients. The mortality of diabetes vs non-diabetes was $7.8 \%$ vs $2.7 \%$, respectively (HR 1.49). Furthermore, the comparison of the mortality for the diabetics between good control group $(7.3 \%$ [6.6-8.2\%]) vs poor control group (8.1\% [7.2-10.1\%]) showed adjusted HR as 0.14 . $^{7}$ These diabetic cases were divided and mortality rates were compared. Former group with max glucose below $180 \mathrm{mg} / \mathrm{dL}$ showed $1.1 \%$ of mortality, whereas latter group with max glucose above $180 \mathrm{mg} /$ dL showed $11.0 \%$. Consequently, even if the case is diabetic, the prognosis may be dependent on the current glycemic control.

Several lockdown policies for COVID-19 were found in the world. Going out from home was prohibited in Italy and Spain, while personal exercises with family were allowed in Germany, France and UK. The Sports Agency of Japan has released a comment for exercise with the cooperation of Japan Medical Association (JMA) ${ }^{8}$ It includes

\section{i. Avoid closed, crowded and close contact,}

ii. Keep a distance for time and place with other people when walking and jogging,

iii. Use videos at home and pay careful attention to ventilation in the room when muscle training and yoga

There was a study to clarify the relationship of leisure-time physical activity (LTPA), total mortality and cardiovascular events. ${ }^{9}$ Subjects were 1702 T2DM patients followed by 8.05 years in median. As a result, Harzard Ratio (HR) was significantly reduced in the top vs bottom tertile of LTPA ( $\geq 15.4$ vs $\leq 3.7$ metabolic equivalents [MET] h/week), which was 0.55 in stroke, 0.49 in total mortality, but not significant of 0.77 in CHD. If people have physical activity 2 times/ week at midlife, they will have decreased risk of dementia (OR 0.48) and Arzheimer disease (AD) (OR 0.38). Thus, regular physical activity would be recommended for risk reduction or delay of dementia and $\mathrm{AD} .^{10}$

Exercise includes aerobic exercise and resistance exercise. Resistance exercise makes muscle fibers thicker, while inactivity or 
sedentary lifestyle reduces muscle fibers thinner. From the age of 60 to 80 , the muscle mass of the thigh generally decreases by $1 \%$ per year. There was an intervention study for reduced activity for 2 weeks. ${ }^{11}$ After healthy elderly people decreased walking steps from 5962 steps to 1413 steps, muscle mass decreases by $3.7 \%$ in 2 weeks. Consequently, only two weeks inactivity may be equivalent to natural decrease of muscle mass for 4 years.

From data of 1.44 million subjects including 187 thousand cancer subjects, the association of leisure-time physical activity and incidence of common cancer was investigated. ${ }^{12}$ There was significant correlation between them, where HR was $0.58-0.79$ in esophageal adenocarcinoma, liver, lung, kidney, gastric cardia, endometrial, and HR was 0.80-0.90 in myeloid leukemia, myeloma, colon, head and neck, rectal, bladder and breast.

Regarding physician's advice to patient for increasing exercise, there was comparative study. ${ }^{13}$ Control arm participants were advised to engage in physical activity $30-60 \mathrm{~min} /$ day. Active arm participants group were advised to increase step by provided pedometers with recorded step counts. As a result, the latter showed a net $20 \%$ or 1200 steps increased step counts associated with $0.38 \%$ decrease of $\mathrm{HbAlc}$.

People should pay attention to the lifestyle with sedentary behavior. ${ }^{14}$ There has been fundamental recommendation for physical activity programs, which is Physical Activity Guidelines for Americans (PAGA). ${ }^{15}$ The figure is introduced using several gradient colors. ${ }^{16}$ It represents all-cause mortality risks; red is higher risk, green is lower risk, orange and yellow are transitional decreases in risk. It is clear that moderate-to-vigorous activity along with reducing sitting time is very beneficial. ${ }^{16}$

There is a comparative exercise study for inactive overweight/ obese T2DM Adults. ${ }^{17}$ Three groups were

i. control as uninterrupted sitting (SIT),

ii. $\quad$ sitting plus 3-min bouts of light-intensity walking (LW) every $30 \mathrm{~min}$, and

iii. sitting plus 3-min bouts of simple resistance activities (SRA) (half-squats, calf raises, gluteal contractions, and knee raises) every $30 \mathrm{~min}$.

Interrupting persisting sitting with brief bouts of LW or SRA can reduce acute postprandial glucose, insulin, C-peptide and triglyceride responses in T2DM adults. With poor adherence to structured exercise, this approach seems to be potentially beneficial and practical. ${ }^{17}$

Combined aerobic and resistance training was investigated for 5 years. ${ }^{18}$ Subjects were 10680 Japanese women, and 166 cases had developed T2DM. By training frequency, subjects were divided into 4 groups, which were $6.3,9.6,12.0,16.0$ times a month, respectively. As lowest frequency group is set to be $1^{\text {st }}$ quartile, hazard ratio (HR) of the risk for developing T2DM was $0.95,0.73$ and 0.69 in $2^{\text {nd }}, 3^{\text {rd }}, 4^{\text {th }}$ quartile, respectively. After adjustment for age, BMI and thigh circumference, HR became $0.84,0.69$, and 0.61 , respectively. Consequently, higher frequency of combined training would bring lower risk for T2DM. ${ }^{18}$

In summary, people's lifestyles are changing due to corona infections. ${ }^{19}$ In the future, middle-aged and diabetic patients should pay attention to inactive daily activities with the belief that exercise has the same power as medicine. Everyone will be expected to continue appropriate health care and self-care.

\section{Funding}

None.

\section{Acknowledgments}

None.

\section{Conflicts of interest}

The author declares that there was no conflict of interest.

\section{References}

1. Hussain YH, Baderkhan B, Hamid M, et al. Mortalities and Morbidities Trends of COVID-19 Infection, From Explosiveness to Aggressiveness, Understanding Gaps in System Response and Transmission Chain Events. $J$ Health Care and Research. 2020;01;1(1):22-27.

2. Lobelo F, Stoutenberg M, Hutber A. The Exercise is Medicine Global Health Initiative: a 2014 update. British Journal of Sports Medicine. 2014;48(22):1627-1633.

3. American College of Sports Medicine. ACSM's guidelines for exercise testing and prescription, $10^{\text {th }}$ ed. USA: LWW; 2017.

4. Bushman BA. Developing the P (for Progression) in a FITT-VP Exercise Prescription. ACSM's Health \& Fitness Journal. 2018;22(3):6-9.

5. Park SW, Goodpaster BH, Lee JS, et al. Excessive Loss of Skeletal Muscle Mass in Older Adults with Type 2 Diabetes. Diabetes Care. 2009;32(11):1993-1997.

6. Liu H, Chen S, Liu M, et al. Comorbid Chronic Diseases are Strongly Correlated with Disease Severity among COVID-19 Patients: A Systematic Review and Meta-Analysis. Aging and Disease. 2020;11(3):668.

7. Zhu L, She ZG, Cheng X, et al. Association of Blood Glucose Control and Outcomes in Patients with COVID-19 and Pre-existing Type 2 Diabetes. Cell Metabolism. 2020;31(6):1068-1077.

8. https://www.mext.go.jp/sports/b menu/sports/mcatetop05/jsa 00010 . html

9. Sone H, Tanaka S, Tanaka S, et al. Leisure-time physical activity is a significant predictor of stroke and total mortality in Japanese patients with type 2 diabetes: analysis from the Japan Diabetes Complications Study (JDCS). Diabetologia. 2013;56(5):1021-1030.

10. Rovio S, Kåreholt I, Helkala EL, et al. Leisure-time physical activity at midlife and the risk of dementia and Alzheimer's disease. Lancet Neurol. 2005;4(11):705-711.

11. Breen L, Stokes KA, Churchward-Venne TA, et al. Two weeks of reduced activity decreases leg lean mass and induces "anabolic resistance" of myofibrillar protein synthesis in healthy elderly. J Clin Endocrinol Metab. 2013;98(6):2604-2612

12. Moore SC, Lee IM, Weiderpass E, et al. Association of Leisure-Time Physical Activity with Risk of 26 Types of Cancer in 1.44 Million Adults. JAMA Intern Med. 2016;176(6):816-825.

13. Dasgupta K, Rosenberg E, Joseph L, et al. Physician step prescription and monitoring to improve ARTERial health (SMARTER): A randomized controlled trial in patients with type 2 diabetes and hypertension. Diabetes Obes Metab. 2017;19(5):695-704.

14. Bushman BA. Physical Activity Guidelines for Americans. ACSM's Health \& Fitness Journal. 2019;23(3):5-9.

15. Office of Disease Prevention and Health Promotion. About the Guidelines. Rockville (MD): Office of Disease Prevention and Health Promotion; 2018 . 
16. U.S. Department of Health and Human Services. Physical Activity Guidelines for Americans, 2nd ed. Washington (DC): U.S. Department of Health and Human Services; 2018.

17. Dempsey PC, Larsen RN, Sethi P, et al. Benefits for Type 2 Diabetes of Interrupting Prolonged Sitting with Brief Bouts of Light Walking or Simple Resistance Activities. Diabetes Care. 2016;39(6):964-972.
18. Sawada SS, Gando Y, Kawakami R, et al. Combined aerobic and resistance training, and incidence of diabetes: A retrospective cohort study in Japanese older women. Journal of Diabetes Investigation. 2019;10(4):997-1003.

19. Bando H. Recommended Hints for Improved Lifestyle and Health from Integrative Medicine (IM) In the Critical Situation of COVID-19. Diab Res Open Access. 2020;2(2):17-21. 